\title{
Wide-field Astronomy with Tech Pan Film - A Positive Outlook for Schmidt Photography
}

\author{
Quentin A Parker \\ Anglo-Australian Observatory, Coonabarabran, NSW 2357, Australia
}

Steven Phillipps

University of Wales, College of Cardiff, Wales, UK

David Morgan

Royal Observatory, Blackford Hill, Edinburgh, Scotland, UK

\begin{abstract}
The introduction of film-based Kodak Technical Pan emulsion at the UKST has led to a new lease of life for deep, wide-field astrophotography. This is because the excellent imaging, resolution and low noise characteristics of Tech Pan typically yield a one magnitude depth gain over equivalent IIIa-F plates. Many new projects are underway to take advantage of the significant information gains. The cheap cost of film and ease of transportation gives direct economic benefits too. Tech Pan could also offer a medium term, competitive alternative to CCD mosaics for providing new, deep, wide-field surveys via stacking of several digitised exposures of the same field. A one magnitude gain could result from the stacking of just 6 Tech Pan films to yield an $R \sim 23.5-24$. Even without digital co-addition the largest current CCDs cannot hope to match Tech Pan's inherent $\sim 3 \mu \mathrm{m}$ resolution and the 40 square degree area coverage of the UKST.
\end{abstract}

\section{Introduction}

Kodak Tech Pan emulsion is an extremely fine grained, pan-chromatic film with extended red sensitivity (Kodak P-255, 1981). Though its astronomical promise was recognised early (Everhard 1981), tests at professional telescopes were discontinued when glass and film samples did not respond well to normal hypersensitisation techniques (West et al. 1981) leading to a prolonged lack of interest among professional astronomers. However, successful UKST tests were carried out in 1991 once hypersensitisation and technical problems associated with film use were solved. Films were obtained which exhibited excellent image quality and resolution and which, in good seeing, went about one magnitude deeper than equivalent IIIa-F emulsion on glass but with considerably lower grain noise. (Russell et al. 1992; Parker 1992). Tech Pan now accounts for $\sim 60$ per cent of non-survey exposures. An exciting variety of astronomical projects are underway to take advantage of the superb imaging, low noise and fainter limits of Tech Pan. 


\section{Basic properties}

Kodak publications P-255 (1981) and P-315 (1987) detail Tech Pan's basic properties; extremely fine, mono-disperse grain ( $\sim 0.5$ that of IIIa emulsions), low rms diffuse granularity and high resolving power $(\sim 320$ lines $/ \mathrm{mm}$ c.f. $\sim 200$ for IIIa emulsions). The panchromatic spectral sensitivity is similar to that of IIIa-F (except for a useful enhancement around $\mathrm{H} \alpha$ ) allowing direct R-band comparisons. The modulation transfer function (MTF) and point spread function (PSF), are also superior and it is capable of a wide contrast range dependant on treatment (Kodak 1987). Finally Tech Pan's emulsion thickness on Estar is only $\sim 11 \mu \mathrm{m}$ c.f. $\sim 20 \mu \mathrm{m}$ for IIIa's.

Sky limited Tech Pan R-band exposures (i.e. $D_{s k y} \sim 1+$ chemical fog) were soon obtained in standard 1 hour exposure times. The gradient of the characteristic curves at sky (the reference $\gamma$ or contrast level) also gave almost identical values to IIIa-F, i.e. $\gamma_{s k y(4415)}=\gamma_{s k y(I I I a-F)}=2.0$ to within $3 \%$ with $\mathrm{N}_{\text {sample }}=50$. The scientific gains are a direct consequence of these basic emulsion properties combined with the success at achieving effective longexposure speed via appropriate hypering, processing and exposure conditions (Parker 1992).

\section{First impressions count. A visual assessment comparison.}

Plates 1a,b show a $2 \times 1.5$ arcmin area from consecutive Tech Pan and IIIa-F exposures taken under survey conditions. Visual inspection of sky limited Rband Tech Pan films reveals much improved image resolution compared with top grade IIIa-F plates The clarity of images and features in extended objects like galaxies (dust lanes etc) was quite stunning. Visual quality control indicated about one magnitude depth gain with typical minimum image diameters estimated to be $\sim 20-25 \mu \mathrm{m}$ cf. 30-35 $\mu \mathrm{m}$ for IIIa's.

Quantitative assessments to put these impressions on a firmer footing were obtained by scanning several films with COSMOS, a fast measuring machine (MacGillivray \& Stobie 1984). The data were compared with IIIa-F data from the same fields (Parker, Morgan \& Phillips 1993). Both direct pixel 'mapping mode' data (MM) and thresholded image-analysis mode (IAM) image parameter data comparisons were made.

\section{Results from COSMOS scans of Tech Pan films}

Tech Pan yields a very uniform sky-background and very low grain noise with sky-background intensity histograms from several film and equivalent IIIa-F plate scans indicating a 3-4 fold reduction in noise to $\sim 0.8 \%$ per pixel. Tech Pan has 4 times as many grains per unit area which are half the size of IIIa emulsion grains. This has a direct bearing on achievable $\mathrm{S} / \mathrm{N}$ near the sky-background following Poisson statistics.

For many applications it is also important to define star/galaxy separation as accurately as possible. COSMOS star/galaxy separation plots, such as Log (image-area) versus isophotal magnitude, reveal improved star/galaxy separation with Tech Pan. Figs 1a,b give examples taken from the Virgo field comparison. The stellar locus is generally extremely well defined for Tech Pan 

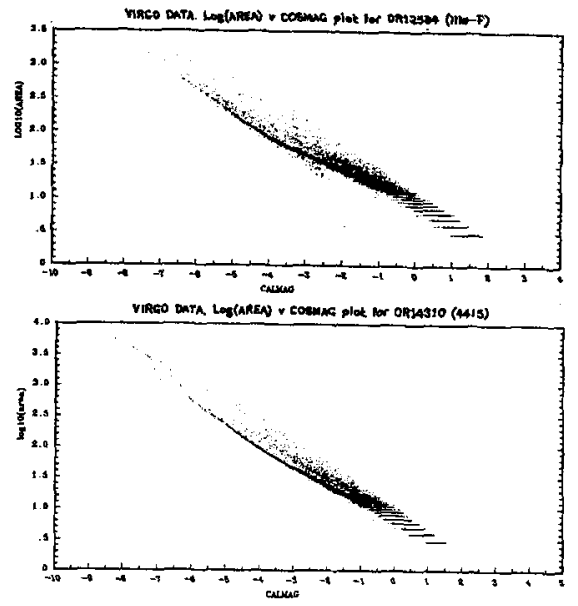

Figure 1. Standard star/galaxy separation plots of Log(image-area) versus COSMOS isophotal magnitudes for the same Virgo cluster area for (a) IIIa-F UKST exposures and (b) Tech Pan.

data allowing separation to fainter magnitudes. Improved deep galaxy catalogues should result.

Number-magnitude counts from Tech Pan and IIIa-F COSMOS data of the same fields confirm the $\sim 1$ magnitude gain for exposures taken under similar conditions (Phillips \& Parker 1993). Low surface brightness (LSB) galaxy profiles obtained from COSMOS MM scans of Tech Pan film can also reach 26.5$27.0 \mathrm{R}$ magnitudes per square arcsec (less than $0.4 \%$ of the sky background) and 1-2 mags better than for IIIa-F. This excellent capability for detecting LSB features at very low isophotal thresholds should enable effective searches for LSB galaxies over large areas, especially if digital co-addition is employed. A number of such projects are already underway, (see Scharwzenberg, Phillipps \& Parker, this meeting).

Magnitude plots of paired images from Tech Pan and IIIa-F COSMOS data gives good $\mathrm{R}$-band magnitude agreement though there is inevitably a small colour term.

\section{A word on $\mathbf{S} / \mathbf{N}$ and DQE}

First DQE estimates of 'optimally' hypered Tech Pan UKST exposures indicated values $\sim 3-4$ times those of IIIa-F, a remarkable result for a photographic emulsion if true. Cannon (1984) described the expected gains if a suitably fast, fine grained emulsion like Tech Pan were to become available. The number of grains in a Tech Pan image near the background is $\sim 4$ times the number in the equivalent image on IIIa-F. This gives a factor two improvement in the linear dimension of the smallest detected extended objects at a given level of surface brightness. Thus if Tech Pan grains are assumed to be equally sensitive and the 
same sky density is reached in the same exposure time as for IIIa-F (as is the case) then Tech Pan's DQE is increased by a factor 4 relative to IIIa-F. Our COSMOS data indeed indicate improvements of a factor 3-4 in the sky-background noise, enabling much lower thresholds to be set.

Now for a photographic emulsion:

$$
D Q E=\left[K \times \gamma^{2} \times(1 / n t)\right] /\left[A \times \sigma_{d}^{2}\right]
$$

where $\mathrm{K}$ is a constant and $\gamma$ is the gradient of the $\mathrm{D}-\log \mathrm{E}$ curve, $n$ is the number of photons per unit area per second and $\sigma_{d}$ is the emulsion RMS diffuse granularity as measured with a scanning aperture A, usually $1000 \mu^{2}$, (e.g. Eccles, Sim \& Tritton 1983). Now we obtain: $\gamma_{4415}=\gamma_{I I I a-F}$ and reach sky in the same exposure time. Hence we can directly relate the DQEs of Tech Pan and IIIa-F. Using the above equation and taking ratios we find:

$$
D Q E_{4415} / D Q E_{I I I a-F}=\sigma_{I I I a-F}^{2} / \sigma_{4415}^{2}
$$

Inserting the known values for $\sigma$ (e.g. Eccles, Sim \& Tritton, 1983) we obtain:

$$
D Q E_{4415}=D Q E_{I I I a-F} \times 3.2 \text {. }
$$

This gives $\mathrm{DQE}_{4415} \sim 10 \%$ if we accept $\mathrm{DQE}_{I I I a-F} \sim 3 \%$, an oft quoted value for hypered IIIa-F (e.g. Kodak 1987).

\subsection{Astrometry from original Tech Pan film exposures.}

Unlike copy film, original film exposures are subjected to deformations at the UKST's focal surface. There have thus been concerns about Tech Pan's ability to mechanically recover and yield good astrometry. To address this, Evans (1993) used APM data to perform an astrometric evaluation of Tech Pan. Results indicate that glass based IIIa emulsions and Tech Pan provide essentially the same accuracy for relative astrometry. Repeat measures of the positions of stars measured from Tech Pan and IIIa-F exposures scanned at both $0^{\circ}$ and $180^{\circ}$ orientation gave $\sigma($ Tech Pan $) \sim 0.04$ arcsec and $\sigma($ IIIa-F) $\sim 0.05$ arcsec. Further studies on a larger set of exposures are underway (Evans, in preparation). These results are not altogether surprising. Kodak Q-34, 1970, shows Estar base to be extremely stable with excellent strength and thermal stability (it is only $\sim 1.8$ times worse than spectroscopic glass plates).

\section{Co-addition of Tech Pan films - An alternative to a CCD mosaic?}

Multi-exposure co-addition has already been used as an effective means of gaining greater depth. The result can be achieved either photographically (Malin 1981) or digitally (Marston 1988; Kemp, 1994). Digital co-addition allows greater control and quantification than photography which is limited by registration difficulties. Computers can also allow for rotation, translation etc in the co-addition process permitting better registration over a full UKST field. The technique is currently restricted by the relatively few exposures available for most UKST fields or to special fields where large numbers of plates already exist (Hawkins these proceedings).

According to Bland-Hawthorn et al. (1993), hereafter BSM93, R band CCD imaging on the UKST with a Loral chip should achieve an $\mathrm{R} \sim 25$ limit at the 
$3 \sigma$ level after $\sim 1000 \mathrm{~s}$ whilst 36 stacked sky-limited IIIa-F exposures can easily reach $\mathrm{R} \sim 24$. Applicability to large areas is impractical with standard IIIa-F plates though as 36 exposures would be required to achieve the 2 magnitude depth gain necessary to compete with CCDs. However, Tech Pan ' $R$ ' exposures already go $\sim 1$ magnitude fainter than existing $R$ survey material (i.e. to $R \sim 23$ ) thus the co-addition of 6 Tech Pan films would gain an extra magnitude to $\mathrm{R} \sim$ 24 (with possible $3 \sigma$ detections to $\sim 25$ ). The relative efficiencies of undertaking deep wide-area surveys with current CCDs, and the digital stacking of IIIa-F and Tech Pan films can be compared. The figures are summarised in Table 1, where the UKST CCD parameters are for a Loral $2048 \times 2048$ chip. The CCD limits refer to $3 \sigma$ detections whilst the photographic limits are conservative though BSM93 claim $3 \sigma$ detections to $\mathrm{R} \sim 25$ from 36 stacked IIIa-F plates.

Table 1. Summary of CCD versus photography at the UKST

\begin{tabular}{|c|c|c|c|c|c|c|}
\hline $\begin{array}{l}\text { Number of } \\
\text { exposures }\end{array}$ & $\begin{array}{l}\text { Depth } \\
\text { in R }\end{array}$ & $\begin{array}{l}\text { Estimated } \\
\text { exp. time }\end{array}$ & $\begin{array}{r}\text { Area } \\
\text { coverage }\end{array}$ & $\begin{array}{r}\text { Inherent } \\
\text { Resolution }\end{array}$ & $\begin{array}{c}\text { Number of } \\
\text { Pixels }\end{array}$ & Telescope \\
\hline $1 \times$ Loral CCD & 25 & $1000 \mathrm{~s}$ & $0.57 \mathrm{sq} . \mathrm{deg}$ & $15 \mu \mathrm{m}$ & $2048 \times 2048$ & UKST \\
\hline$\sim 160 \times$ Loral CCD & 25 & $25 \mathrm{hr}$ & 40 sq.deg & $15 \mu \mathrm{m}$ & $6.7 \times 10^{8}$ & UKST \\
\hline $36 \times 111 a-F$ & 24 & $36 \mathrm{hr}$ & 40 sq.deg & $5 \mu \mathrm{m}$ & $1.8 \times 10^{11}$ & UKST \\
\hline $6 \times 4415$ & 24 & $6 \mathrm{hr}$ & 40 sq.deg & $\sim 3 \mu \mathrm{m}$ & $8.4 \times 10^{10}$ & UKST \\
\hline $1 \times$ IIIa-F & 22 & $1 \mathrm{hr}$ & 40 sq.deg & $5 \mu \mathrm{m}$ & $5 \times 10^{9}$ & UKST \\
\hline $1 \times 4415$ & 23 & $1 \mathrm{hr}$ & 40 sq.deg & $\sim 3 \mu \mathrm{m}$ & $1.4 \times 10^{10}$ & UKST \\
\hline $1 \times \mathrm{RCA} \mathrm{CCD}$ & 24 & $300 \mathrm{~s}$ & 0.05 sq.deg & $\sim 30 \mu \mathrm{m}$ & $512 \times 320$ & AAT \\
\hline
\end{tabular}

Given the ease of transportation of Tech Pan film and the commissioning of the next generation of measuring machine, SuperCOSMOS (Miller et al. 1992), this technique could be a cost-effective medium term alternative to a UKST CCD mosaic for performing deep, large-area R-band surveys.

\section{Uses of Tech Pan at the UKST and future projects.}

New prospects for projects with Tech Pan should exploit its excellent imaging, resolution and low noise characterstics. A major role would be as a superior replacement for IIIa-F for R-band imaging. Digital co-addition of multiple exposures of the same field could also be important.

Tech Pan has useful blue sensitivity too so that use of the UG1 ' $U$ ' filter enables satisfactory U-band imaging with no significant red-leak detected. No loss in depth compared with IIIa-J U plates is evident whilst all the advantages of improved resolution etc are available (Parker, Morgan \& Phillipps 1993).

Tech Pan is also an effective alternative to IIIa-F for objective-prism exposures. The spectral sensitivity of Tech Pan, being somewhat flatter than IIIa-F's, is better for high redshift QSO searches since the bumps in the IIIa-F spectral sensitivity can mimick QSO emission lines, preventing proper exploitation in this area. Preliminary results (Drinkwater 1993) indicate that the improved resolution and $\mathrm{S} / \mathrm{N}$ do enable better feature discrimination. Objective-prism studies of the Magellanic clouds for planetary nebulae, carbon stars etc would also benefit from the greater $\mathrm{S} / \mathrm{N}$ and resolution in the Tech $\mathrm{Pan}$ prism spectra.

The useful sensitivity peak of Tech Pan around $\mathrm{H} \alpha$ indicates that it could replace the coarse grained 098 and the IIIa-F emulsion for $\mathrm{H} \alpha$ narrow-band 

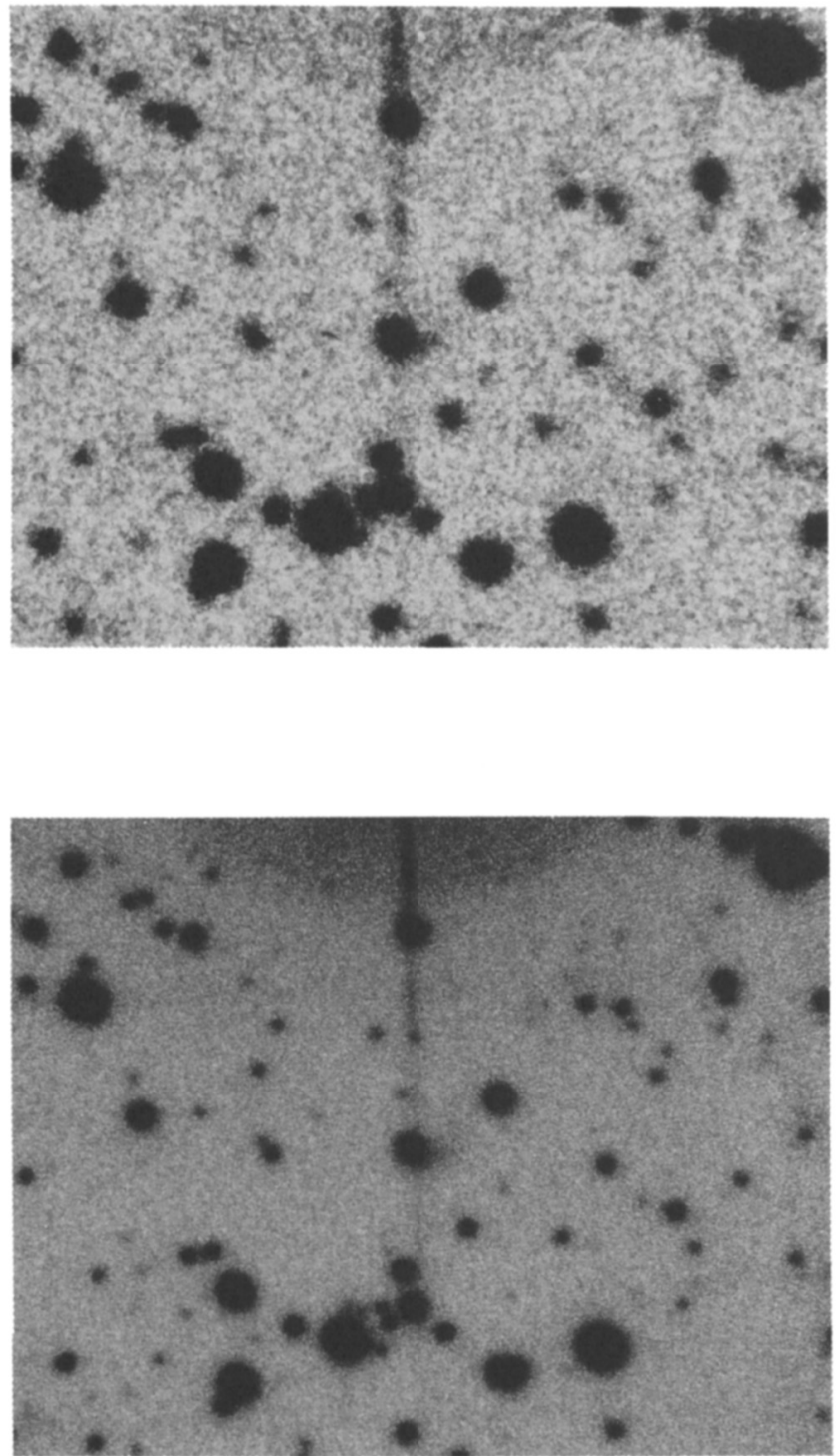

Plates 1a,b Direct IIIa-F (above) to Tech Pan (below) image comparison from consecutive UKST exposures of the same survey field (field 263) taken under survey conditions. 
imaging. COSMOS MM data from two $\mathrm{H} \alpha$ exposures of the same field indicate that the same depth is being achieved as with IIIa-F.

Finally, film provides a cheap means of follow-up exposures of transient phenomena, comets, fast moving asteroids, novae etc.

\section{The main advantages \& disadvantages of Tech Pan}

The fine grain and improved DQE from effective hypering and processing leads to better image quality, improved resolution, fainter isophotal limits, higher photometric accuracy, fainter magnitude limits and better detection of low surface brightness features. The reported scientific gains are the consequence. The cost of Tech Pan films is only 10\% that of glass plates and the film has additional transportation, storage and handling benefits. The estar-base itself is extremely stable and strong (Kodak publication Q-34, 1977) and unlike glass plates can't be broken. Finally the hypered product stores extremely well in an inert atmosphere in cold storage with no apparent degradation in fog levels with long ( 1 month) storage times (Parker 1992).

Tech Pan film was proving difficult to copy though the problems have now largely been solved (Hadley, private communication). Film also needs careful mounting for proper scanning with a measuring machine such as SuperCOSMOS. Film tends to pick up dust easily (being more prone to static) and so needs proper storage and cleaning. Longer development times are required for Tech Pan as push-processing is used. The film can also 'kink' fairly easily making subsequent copying or machine scanning difficult so it still requires careful handling. Finally more halation effects are seen with bright images than with IIIa-F.

\section{References}

Bland-Hawthorn J., Shopbell P. L. \& Malin D.F., 1993, AJ, 106, 2154

Cannon R. D., 1984, In "Astronomical Photography", p119, Occasional reports of the Royal Observatory Edinburgh, eds M. E. Sim \& K. Ishida.

Drinkwater M., 1993, AAO Newsletter 64

Eccles M. J., Sim, M. E., \& Tritton K. P., 1983, in Low level light detectors in Astronomy, chapters $2 \& 3$, Cambridge University press

Evans D. W., 1993, in IAU Commission No.9, Newsletter No.3, p64

Everhard E., 1981, in Astronomical Photography, p117., Occasional reports of the Royal Observatory Edinburgh, eds J. L. Heudier \& M. E. Sim

Kemp S. N., 1994, A\&AS, 282, 425

Kodak Publication Q-34, 1970, Dimensional stability of KODAK Estar base films for the Graphic arts

Kodak Publn.P-255, 1981, KODAK Technical Pan Film

Kodak Publn.P-315, 1987, Scientific Imaging with KODAK films

MacGillivray H. T. \& Stobie R. S., 1984, Vistas in Astronomy, 27, 433

Malin D. F., 1981, J. Photog. Sci. 21, 199

Marston A. P., 1988, MNRAS, 255, 295

Miller L., Cormack W., Paterson M. J., Beard S. M. \& Lawrence L., 1992, in Digitised Optical Sky Surveys II, p133, Kluwer academic publishers. 
Parker Q. A., 1992, Report on Kodak Tech Pan $\$ 415$ estar-based emulsion, AAO internal document

Parker Q. A., Morgan D. H. \& Phillipps S., 1993, IAU Commission No.9, Newsletter No.3, p60

Phillips S. \& Parker Q. A., 1993, MNRAS, 265,385

Russell K. S., Malin D. F., Savage A., Hartley M. \& Parker Q. A., 1992, in 'Digitised Optical Sky Surveys II, p23, Kluwer academic publishers

West R. M., Kurtanidze O.M., Geonijan L.A. \& Kimeridze G. N., 1981, AAS Photo-Bulletin No 28, p3

\section{Discussion}

Lasker: I share your optimism that there will someday be a blue-sensitized Tech Pan, say 4415J. However, what if this doesn't happen? Have you considered a practically achievable blue filter? If so, what might it be?

Parker: I am not optimistic that a blue-sensitized Tech Pan emulsion will be made available by Kodak in the medium term, given the current Kodak problems. However a blue sensitive Tech Pan emulsion would be extremely valuable so this should be pursued vigorously! Manufacture of a suitable filter to isolate an approximation to the B-band from the panchromatic Tech Pan sensitisation has been considered. This is an expensive option due to the composite nature of such a filter. It is difficult to produce a uniform $14 \times 14 \operatorname{arcsec}^{2}$ filter with the desired optical properties.

Murray: Can you say more about the astrometric performance of Tech Pan film? Specifically, how accurately can one map two exposures on the same field on to each other?

Parker: I have only performed crude comparisons based on COSMOS data which indicate consistency between COSMOS scans on two different Tech Pan films of the same field to better than 0.5 arcsec in $\alpha, \delta$. Dafydd Evans at RGO has performed more stringent astrometric tests and I urge you to contact him for details. His preliminary results are encouraging as he finds that the Tech Pan film yields results 'as good as and in some ways better' than from glass plates. From averaged positions of stars on plates and films (from $0^{\circ}$ and $180^{\circ}$ scans) he found $\sigma_{f i l m}=0.027^{\prime \prime}$, and $\sigma_{\text {plate }}=0.026^{\prime \prime}$. 